\title{
Capital Accumulation in an Economy with Heterogeneous Agents and Moral Hazard
}

\author{
Radim Boháček* \\ CERGE-EI, Prague, Czech Republic
}

January 2, 2001

\begin{abstract}
This paper studies a closed economy with a continuum of agents and moral hazard. Economic agents in the economy operate a stochastic production technology with capital and labor inputs in which the latter is private information. I characterize efficient allocations of capital, labor, and consumption in a stationary recursive equilibrium for a decentralized economy with component planners. Allocation and accumulation of capital are facilitated by a 'capital planner' who serves as a financial intermediary for the component planners. In equilibrium, private information lowers the equilibrium interest rate below agents' discount rate and I show that contrary to the private-information endowment economies, a moral-hazard productive economy can exhibit both endogenous lower and upper bounds on the stationary distribution of utility entitlements.

JEL Classification: C68, D31, D82, E10
\end{abstract}

*I am grateful to Pierre-André Chiappori, Daniel Fernandez, José Ignacio Sánchez-Macías, Joao Rato, Thomas J. Sargent, Nancy L. Stokey, the members of the Money and Banking and Theory Lunch Workshops, and especially to Fernando Alvarez, Robert E. Lucas, Jr., Edward C. Prescott, Robert M. Townsend, Carlos Pérez-Verdía, and the anonymous referees for suggestions and criticism. Natalia Kovrizhnyh provided an excellent research assistance. All errors are mine. Email: radim.bohacek@cerge.cuni.cz 


\section{Introduction}

This paper studies a general equilibrium model of a closed economy with heterogeneous agents and moral hazard. The agents operate independent stochastic production technologies with capital and labor inputs in which the latter is private information. I characterize the information-constrained optimal allocations of resources and the conditions for which a stationary equilibrium in a closed economy exhibits an endogenous interest rate with capital accumulation and an invariant, non-degenerate distribution of resources and utility entitlements.

The first contribution of this paper lies in its incorporation of a general production technology into the private-information, moral hazard economy with heterogeneous agents. I decentralize the economy by using the concept of component planners who trade capital among themselves according to the willingness of their agents to supply labor effort. The component planners rent capital from a financial intermediary called 'capital planner' at an endogenously determined marketclearing interest rate. As the surplus of each component planner in each period is invested at the capital planner as the next period capital stock, the capital planner also facilitates capital accumulation. Previous models with private information and capital accumulation have imposed severe restrictions on preferences and technology (as in Khan and Ravikumar (1997a) or Khan and Ravikumar (1997b)). In my decentralized economy with capital planner, not only are all variables endogenous but there are also no special restrictions on preferences, production technology, information structure, or contracts. I confirm the finding of Kehoe and Levine (1993) or Atkeson and Lucas (1995) and show that moral hazard also lowers the equilibrium interest rate below the discount rate of the agents.

Second, I prove that a moral-hazard production economy can exhibit endogenous lower and upper bound on its stationary distribution of utility entitlements. This feature contrasts with the necessity of imposing an exogenous lower bound on utility entitlements as in Thomas and Worrall (1990), Atkeson and Lucas (1992), or Phelan (1995) who motivate the exogenous lower bound by the inability of the agents to forego future consumption or by competition among planners. 
My arguments extend the findings of Aiyagari and Alvarez (1995) who introduce the concept of 'misery', the lowest available utility forever. If misery is not incentive compatible, the distribution of utility entitlements is non-degenerate as far as the consumption set is compact and the technology sufficiently productive to pay for the input costs and consumption of the agent. Moreover, if the planners' cost function is decreasing at the lowest levels of utility entitlements, the lower bound on the stationary distribution will be endogenous: the Pareto-improving policy is to move to the non-decreasing part of the cost function where the agent is better off and the planner incurs a lower cost. The endogenous upper bound follows from the planners' intertemporal tradeoff of resources at an interest rate lower than the agent's discount rate. Thus the ergodic set in a moral hazard production economy can be quite different from those in economies with an endowment technology or truth-telling constraints. Numerical simulations in Bohacek (2000) confirm these analytical results.

The paper analyzes allocations and distribution of resources in a dynamic, general equilibrium model of a closed economy with many agents and moral hazard. It builds on the seminal contributions of Atkeson and Lucas (1992, 1995), Aiyagari and Alvarez (1995) and the growing literature on computable general equilibrium models with heterogeneous agents. The other important stream of the literature has focused on partial equilibrium principal-agent models as in Green (1987), Spear and Srivastava (1987), Atkeson (1991), Phelan and Townsend (1991), or Lehnert, Ligon, and Townsend (1998). In these models the principal usually has access to outside funds at an exogenously given price.

The paper is organized as follows. In the next Section, I describe preferences, production technology, the private information economy, and outline a social planner's problem. In Section 3, I follow Atkeson and Lucas (1995) and decentralize the economy using the concept of component planners and a capital planner. I formulate the problem recursively and define a stationary recursive equilibrium. Optimal allocations, ergodic sets, properties of the invariant distribution, and an algorithm for numerical simulation are characterized in Section 4. Section 5 concludes. All mathematical proofs are in the Appendix. 


\section{A Social Planner's Problem}

In this section, I formally describe the economy, state the assumptions on preferences and production function, commodity space, and information structure. Then I present the economy as a principal-agent problem with moral hazard.

The closed economy is populated by a continuum of agents of measure one. In each period of time $t=0,1,2, \ldots$, each agent is endowed with one unit of time he can divide into labor, $l_{t}$, and leisure, $1-l_{t}$. At the beginning of each period, an agent chooses labor supply to work with his capital stock, $k_{t}$, accumulated over time from an initial positive stock $k_{0}$, in his production technology $f\left(k_{t}, l_{t}\right)$. At the end of the period, he divides the realized output, $y_{t}$, into consumption, $c_{t}$, and investment towards the next period capital stock, $k_{t+1}$, which depreciates at a rate $\delta \in(0,1)$. Each period the agent derives a separable utility from consumption and leisure, $u\left(c_{t}\right)+v\left(1-l_{t}\right)$, discounted over time at $\beta \in(0,1)$.

Assumption 1 The production function $f: \mathbb{R}_{+} \times[0,1] \rightarrow \mathbb{R}_{+}$is concave, continuously differentiable, and homogeneous of degree one. $f(0, l)=f(k, 0)=0$ and for $x=\{k, l\}, \lim _{x \rightarrow \infty} f_{x}^{\prime}(k, l)=0$ and $\lim _{x \rightarrow 0} f_{x}^{\prime}(k, l)=\infty$.

With decreasing marginal productivity of capital, there exists a maximal (golden-rule) finite level of capital stock maintained by each agent in the steady state when labor supply is at its highest level, $l=1$. Denoting this level by $\bar{k}$ and the set of capital stock by $K=[0, \bar{k}]$, the steady-state set of output is $Y=[0, \bar{y}]$ for $\bar{y}=f(\bar{k}, 1)$. Finally, with consumption $c \in C=[0, \bar{c}]$, where $\bar{c}=\bar{y}-\delta \bar{k}<\infty$ is the maximal sustainable consumption in a steady state, all sets are compact.

Assumption 2 The functions $u:[0, \bar{c}] \rightarrow \mathbb{R}$ and $v:[0,1] \rightarrow \mathbb{R}$ are twice continuously differentiable, strictly concave, strictly increasing in $c$ and decreasing in $l$, and bounded from above and below.

Agent's labor supply and capital input are related to output realizations by an exogenous probability measure $P$ on Borel measurable space $(Y, \mathcal{B}(Y))$ where $\mathcal{B}(Y)$ denotes Borelians on $Y$. The number $P(A \mid k, l)$ represents the probability of 
output $y$ being an element of $A \in \mathcal{B}(Y)$ given the inputs $k \in K$ and $l \in L$ were employed in the production function.

Assumption 3 For each $(k, l) \in(0, \bar{k}] \times(0,1]$, the probability $P(A \mid k, l)$ is strictly positive for all $A \in \mathcal{B}(Y)$.

The above assumption establishes the information asymmetry: other agents or a social planner cannot infer with certainty an agent's labor supply from an observed positive output (except for a zero recommended input resulting in zero output with probability one). The output realizations drawn from this stochastic technology are independent in time and across agents. Except for the monotone likelihood ratio condition there are no other restrictions on the probability distribution known to all agents in the economy.

The problem of each agent is to maximize his lifetime utility subject to his budget constraint. Each agent can only employ his own labor but can trade capital and consumption goods with other agents. Due to the stochastic technology, the risk-averse agents would like to write insurance contracts with each other to smooth their consumption. I will model the economy as a principal-agent problem for a social planner with heterogeneous agents in a closed economy. It is assumed that the planner has the ability to prevent the agents from trading with each other.

In exchange for providing the agents with insurance, the social planner confiscates the initial capital stock and assigns infinite sequences of allocations to each agent contingent on an agent's identification and history of output realizations. At each date $t \geq 0$, the social planner identifies each agent by an initial entitlement to expected, discounted utility $w_{0} \in W=[\underline{w}, \bar{w}] \subset \mathbb{R} .^{1}$ All agents identified with the same $w_{0}$ receive the same treatment. Let $\psi_{0}$ denote the distribution of initial expected utility entitlements on $(W, \mathcal{B}(W))$ so that $\psi_{0}(A)$ represents a fraction of the population entitled to expected discounted utility in $A \in \mathcal{B}(W)$.

The realizations of each agent's stochastic technology form a history of output $y^{t}=\left(y_{0}, y_{1}, \ldots, y_{t}\right) \in Y^{t+1}=Y_{0} \times Y_{1} \ldots \times Y_{t}$. Then at date $t \geq 0$, an agent

\footnotetext{
${ }^{1}$ I will discuss in detail the values of $\{\underline{w}, \bar{w}\}$ in Section 4 when analyzing the endogenous bounds of the stationary distribution.
} 
$w_{0}$ 's consumption is a sequence of functions $c_{t}: W \times Y^{t+1} \rightarrow[0, \bar{c}]$, and similarly for labor input, $l_{t}: W \times Y^{t} \rightarrow[0,1]$, and capital input, $k_{t}: W \times Y^{t} \rightarrow[0, \bar{k}]$. Denote the histories of these functions also with superscripts and define $P^{t+1}$ as probability assigned to an output history $y^{t} \in Y^{t+1}$ given a history of capital and labor inputs,

$$
\begin{aligned}
P^{t+1}\left(y^{t} \mid k^{t}\left(w_{0}, y^{t-1}\right), l^{t}\left(w_{0}, y^{t-1}\right)\right)= & P\left(y_{0} \mid k_{0}\left(w_{0}\right), l_{0}\left(w_{0}\right)\right) \cdot \\
& \cdot P\left(y_{1} \mid k_{1}\left(w_{0}, y^{0}\right), l_{1}\left(w_{0}, y^{0}\right)\right) \cdot \ldots \\
& \cdot P\left(y_{t} \mid k_{t}\left(w_{0}, y^{t-1}\right), l_{t}\left(w_{0}, y^{t-1}\right)\right) .
\end{aligned}
$$

Within this framework, at time $t \geq 0$ the social planner assigns to an agent identified by $w_{0} \in W$ an allocation sequence

$$
\sigma=\left\{k_{t}\left(w_{0}, y^{t-1}\right), l_{t}\left(w_{0}, y^{t-1}\right), c_{t}\left(w_{0}, y^{t}\right)\right\}_{t=0}^{\infty}
$$

so that the latter's expected initial discounted utility can be written as

$$
\begin{aligned}
U\left(w_{0}, \sigma\right)=\sum_{t=0}^{\infty} \beta^{t} \int_{Y^{t+1}}\left\{u\left(c_{t}\left(w_{0}, y^{t}\right)\right)\right. & \left.+v\left(1-l_{t}\left(w_{0}, y^{t-1}\right)\right)\right\} \cdot \\
\cdot & P^{t+1}\left(d y^{t} \mid k^{t}\left(w_{0}, y^{t-1}\right), l^{t}\left(w_{0}, y^{t-1}\right)\right) .
\end{aligned}
$$

Define an allocation as such a sequence $\sigma$ from the set of all sequences that satisfies the following restrictions: first, the promise keeping constraint requiring the sequence $\sigma$ to deliver the initial expected discounted utility entitlement,

$$
w_{0}=U\left(w_{0}, \sigma\right)
$$

for all $w_{0} \in W$. Second, because of the moral hazard problem, the sequence must be incentive compatible,

$$
U\left(w_{0}, \sigma\right) \geq U\left(w_{0}, \hat{\sigma}\right)
$$

for all $w_{0} \in W$, where $\hat{\sigma}$ contains any labor supply deviation $\hat{l} \in L$ from the recommended $l \in L$ in any period of time.

Since the economy is populated by a continuum of agents, at the beginning of each period, the social planner is required to divide the whole aggregate capital 
stock into capital input assignments for all agents $w_{0} \in W$,

$$
\bar{K}_{t} \equiv \int_{W \times Y^{t}} k_{t}\left(w_{0}, y^{t-1}\right) P^{t}\left(d y^{t-1} \mid k^{t-1}\left(w_{0}, y^{t-2}\right), l^{t-1}\left(w_{0}, y^{t-2}\right)\right) \psi_{0}\left(d w_{0}\right) .
$$

Finally, the allocations of all agents must be feasible in each time period,

$$
\begin{gathered}
\int_{W \times Y^{t+1}}\left\{y_{t}-c_{t}\left(w_{0}, y^{t}\right)\right\} P^{t+1}\left(d y^{t} \mid k^{t}\left(w_{0}, y^{t-1}\right), l^{t}\left(w_{0}, y^{t-1}\right)\right) \psi_{0}\left(d w_{0}\right) \\
\geq \bar{K}_{t+1}-(1-\delta) \bar{K}_{t} .
\end{gathered}
$$

This equation also serves as the law of motion for the capital stock: all goods produced but not consumed by the agents are added by the social planner to the depreciated current capital stock.

The rest of the paper is devoted to characterization of efficient allocations and distribution of utility entitlements in a stationary recursive equilibrium for a decentralized economy with component planners and a financial intermediary.

\section{A Decentralized Recursive Formulation}

Following Atkeson and Lucas (1995), the problem of finding the efficient allocations can be partially decentralized by using prices and the concept of 'component planners', each responsible for allocating resources only to agents entitled to an initial utility entitlement $w_{0}$. Each component planner chooses an allocation that attains the utility entitlement of his subpopulation in such a way as to minimize the cost of attaining $w_{0}$ evaluated at prices he trades with the other component planners.

Specifically, the component planners borrow and lend capital inputs according to the willingness of their agents to supply labor effort. One can imagine the capital trading intermediated by a zero-profit financial intermediary called the "capital planner'. All component planners deposit their initial capital stocks of their agents with the capital planner who then lends the capital back to the component planners in each period at a market-clearing price $r_{t}+\delta$. In other words, the capital planner announces a sequence of interest rates $\left\{r_{t}\right\}_{t=0}^{\infty}$ such that the gross-of-depreciation 
rate of return on capital is equal across the component planners and the whole capital stock is lent out in each period. All loans are repaid at the end of each period and the component planners invest at the capital planner all goods produced but not consumed by the agents as the next-period aggregate capital stock.

Given the interest rate sequence $\left\{r_{t}\right\}_{t=0}^{\infty}$, a component planner assigned to subpopulation $w_{0} \in W$ chooses an allocation sequence $\sigma_{r}$ such that it minimizes the cost of resources

$$
\begin{aligned}
& \int_{Y_{0}}\left\{c_{0}\left(w_{0}, y_{0}\right)+\left(r_{0}+\delta\right) k_{0}\left(w_{0}\right)-y_{0}\right\} P\left(d y_{0} \mid k_{0}\left(w_{0}\right), l_{0}\left(w_{0}\right)\right)+ \\
&+\sum_{t=1}^{\infty} \prod_{s=0}^{t-1} \frac{1}{1+r_{s}} \int_{Y^{t+1}}\left\{c_{t}\left(w_{0}, y^{t}\right)+\left(r_{t}+\delta\right) k_{t}\left(w_{0}, y^{t-1}\right)-y_{t}\right\} \\
& \cdot P^{t+1}\left(d y^{t} \mid k^{t}\left(w_{0}, y^{t-1}\right), l^{t}\left(w_{0}, y^{t-1}\right)\right)
\end{aligned}
$$

subject to the promise keeping (1) and the incentive compatibility (2) constraints. Again, the capital stock must be distributed between agents in each period and allocations of all component planners must be feasible in the closed economy. It is straightforward to apply the First Welfare Theorem as in Atkeson and Lucas (1992) in order to establish the efficiency outcome of the component planning problem.

There is no general method of solving for the whole interest rate sequence $\left\{r_{t}\right\}_{t=0}^{\infty}$ that would clear the market among the component planners. However, a steady state of the decentralized economy will exhibit a constant interest rate $r$ at which the optimal allocation policies $\sigma_{r}$ lead to an invariant distribution of utility entitlements $\psi_{r}$ that satisfy the market-clearing conditions for a closed economy with constant aggregate capital stock $\bar{K}_{r}$.

In the recursive formulation the state variable of each component planner is only the utility entitlement $w$ that summarizes the history of output realizations of his subpopulation at the beginning of each period. An agent is now assigned inputs $k(w)$ and $l(w)$ as functions of the current utility entitlement, while consumption $c(w, y)$ and the continuation utility entitlement $w^{\prime}(w, y)$, the next-period state variable, are also contingent on the realization of output. 
In the steady state, the capital planner rents the accumulated stock of capital $\bar{K}_{r}$ to the component planners at a constant interest rate $r$. Each component planner borrows an efficient amount of capital input according to his agent's willingness to supply labor input. At the end of each period, all component planners pay for their capital loans $(r+\delta) k(w)$ and deposit all remaining surplus $y-c(w, y)$ at the capital planner: in the steady state, the aggregate surplus exactly equals the depreciated capital stock $\delta \bar{K}_{r}$.

Therefore, for a constant interest rate $r$, define an allocation policy of a component planner associated with subpopulation $w \in W$ as

$$
\sigma_{r} \equiv\left\{k(w), l(w), c(w, y), w^{\prime}(w, y)\right\}
$$

where $k: W \rightarrow[0, \bar{k}], l: W \rightarrow[0,1], c: W \times Y \rightarrow[0, \bar{c}]$, and $w^{\prime}: W \times Y \rightarrow \mathbb{R}$. The objective of each component planner is to minimize the present value of resources needed to provide an honest agent with a lifetime utility $w$ at interest rate $r$. For intertemporal price of resources $1 /(1+r)$ and all $w \in W$, define a value function $V_{r}: W \rightarrow \mathbb{R}$ for the component planning problem and an operator $T_{r}$ on the space of bounded, continuous functions $D(W)$ as

$$
\begin{aligned}
& \quad\left(T_{r} V_{r}\right)(w)= \\
& \inf _{\sigma_{r}} \int_{Y}\left\{c(w, y)+(r+\delta) k(w)-y+\frac{1}{1+r} V_{r}\left(w^{\prime}(w, y)\right)\right\} P(d y \mid k(w), l(w)),
\end{aligned}
$$

subject to the promise keeping constraint,

$$
w=\int_{Y}\left\{u(c(w, y))+v(1-l(w))+\beta w^{\prime}(w, y)\right\} P(d y \mid k(w), l(w)),
$$

and the incentive constraint for all $(l, \hat{l}) \in L \times L$,

$$
\begin{aligned}
\int_{Y}\{u(c(w, y)) & \left.+v(1-l(w))+\beta w^{\prime}(w, y)\right\} P(d y \mid k(w), l(w)) \\
& \geq \int_{Y}\left\{u(c(w, y))+v(1-\hat{l})+\beta w^{\prime}(w, y)\right\} P(d y \mid k(w), \hat{l}),
\end{aligned}
$$

where $\hat{l} \in L$ represents any deviation from the recommended labor effort.

In a stationary recursive equilibrium, the allocation of all individual component planners must lead to an invariant distribution of utility entitlements. For that 
purpose define a probability measure $\lambda_{r}$ from the set $\Lambda$ of all probability measures on $(W, \mathcal{B}(W))$, and a transition function $F_{r}: W \times \mathcal{B}(W) \rightarrow[0,1]$. With $F_{r}(w, A)$ representing a probability that an agent with a current utility entitlement $w \in W$ will be entitled to $w^{\prime}$ belonging to $A \in \mathcal{B}(W)$ next period, the probability measure $\lambda_{r} \in \Lambda(W, \mathcal{B}(W))$ is invariant provided that

$$
\lambda_{r}(A)=\int_{W} F_{r}(w, A) \lambda_{r}(d w) \quad \text { for all } A \in \mathcal{B}(W) .
$$

Using the invariant probability measure, the aggregate stock of capital is constant,

$$
\bar{K}_{r} \equiv \int_{W} k(w) \lambda_{r}(d w)
$$

and the market clearing condition requires that the allocation policies of all component planners are feasible in the closed economy with constant aggregate capital stock,

$$
\int_{W \times Y}\{y-c(w, y)\} P(d y \mid k(w), l(w)) \lambda_{r}(d w)=\delta \bar{K}_{r} .
$$

It is now possible to state the definition of a stationary recursive equilibrium.

Definition 1 A stationary recursive equilibrium for the decentralized economy is a constant interest rate $r$, a value function $V_{r}$, an allocation policy $\sigma_{r}$, a probability measure $\lambda_{r}$, and a law of motion for aggregate capital stock $\bar{K}_{r}$, such that

1. at interest rate $r$, for all $w \in W$, the allocation policy $\sigma_{r}$ minimizes the objective function of each component planner (5) subject to the constraints (6) and (7);

2. the probability measure $\lambda_{r}$ is invariant (8);

3. the aggregate capital stock (9) is constant with $\bar{K}_{r}<\infty$;

4. and the market-clearing condition (10) holds.

Finally, it follows from Theorems 9.2 in Stokey, Lucas, and Prescott (1989) that at a constant interest rate, the optimal allocations of the recursive and sequential formulations are equivalent. 


\section{Characterization of Optimal Allocations}

In this section I study the existence, uniqueness as well as the properties of the optimal allocation and the stationary distribution of utility entitlements in the steady state of the decentralized economy. All proofs are in the Appendix.

In general, models with moral hazard exhibit non-convexity of the constraint set for the component planner's problem. This property can be regained by lotteries as in Phelan and Townsend (1991). With lotteries, the choice of a component planner associated with agents entitled to $w \in W$ is a probability measure $\pi\left(k, l, y, c, w^{\prime} \mid w\right)$ over all points in $K \times L \times Y \times C \times W$. Following Prescott and Townsend (1984), I show that conditional on recommended inputs and output realization, the probability measure $\pi\left(c, w^{\prime} \mid k, l, y ; w\right)$ puts all mass on a single point in the consumption and continuation utility entitlement set.

Proposition 1 With lotteries, the operator $T_{r}$ has a unique fixed point $V_{r}$ in the space of bounded, continuous functions $D(W)$ and for all $V \in D(W)$, $\lim _{n \rightarrow \infty} T_{r}^{n} V \rightarrow V_{r}$. For a strictly convex $V \in D(W)$ and all $w \in W$, the minimum of the component planner's problem is attained by a unique continuous policy function $\pi$. Conditional on $(k, l, y) \in K \times L \times Y$ there exists a pair of $\left(c, w^{\prime}\right) \in C \times W$ such that the optimal policy $\pi\left(c, w^{\prime} \mid k, l, y ; w\right)=1$ for every $w \in W$.

Thus for a strictly convex value function, the minimum of the component planner problem is attained by unique continuous policy functions $c(w, y)$ and $w^{\prime}(w, y)$ for each input choice, $k(w)$ and $l(w)$, and a realized output, $y$. This result allows me to take the first order conditions with respect to consumption and continuation utility entitlements which are both important for studying the existence and properties of the stationary recursive equilibrium.

Lemma 1 For all $w \in W$,

1. $w^{\prime}(w, y)$ and $c(w, y)$ are increasing functions of $w$ for all $y \in Y$;

2. $w^{\prime}(w, y)$ and $c(w, y)$ are increasing functions of $y$ for all $l \in(0,1]$. 
That consumption and the continuation utility entitlement are increasing functions of the current utility entitlement and that agents are rewarded for a high output realization (except for $l=0$ when the allocations are independent of output) are standard results of the private information literature. With continuation utility entitlements increasing (decreasing) for agents who realized high (low) output, there is a need to study the existence and properties of the ergodic set for the invariant distribution of utility entitlements.

Recall that Green (1987) and Thomas and Worrall (1990) established that without a lower bound the utility entitlements converge to minus infinity with probability one. Following the result of Aiyagari and Alvarez (1995), I assume that the consumption set is compact, $c \in[0, \bar{c}]$, where $\bar{c}<\infty$, and show that it is not incentive compatible to drive an agent to the lower bound of this consumption set: an agent offered a zero consumption forever cannot be motivated to supply positive labor effort. It follows that the stationary distribution of utility entitlements will have at least this exogenous lower bound at the utility entitlement corresponding to repeated zero consumption and full leisure.

Next, the stationary distribution of utility entitlements is non-degenerate provided there exists an incentive compatible positive labor supply for which the technology is productive enough to pay for the capital input and current consumption of the agent. Then the cost-minimizing component planners assign positive labor supply and reward their agents by an increased next-period utility entitlement for a high output realization (and vice versa for a low one).

Moreover, if the planners' cost function is decreasing at the lowest levels of utility entitlements (which implies that incentive-compatible labor supply and expected output are increasing), both planner and agent are better off by moving to the Pareto frontier in the non-decreasing part of the cost function: the distribution of utility entitlements has an endogenous lower bound $w_{*}$ where the convex cost function has its minimum. Endogeneity of the upper bound $w^{*}$ follows from the intertemporal tradeoff of resources: its existence is guaranteed for an interest rate lower than the agent's discount rate.

In order to study the existence of lower and upper bounds on the stationary 
distribution of utility entitlements, it is convenient to set the (non-binding) exogenous upper bound at a utility entitlement corresponding to a perpetual utility from full leisure and the highest consumption, $\bar{w} \equiv(1-\beta)^{-1}\{u(\bar{c})+v(1)\}$, where $\bar{c}=\bar{y}-\delta \bar{k}<\infty$ is the maximal sustainable consumption in a steady state.

Second, define $w_{m}$ as forever repeated assignment of zero consumption and zero leisure, $w_{m} \equiv(1-\beta)^{-1}\{u(0)+v(0)\}$. Aiyagari and Alvarez (1995) call this utility entitlement 'misery' and show that if misery is not incentive compatible, there is a non-degenerate distribution of utility entitlements in a steady state. It is obvious that an agent assigned zero consumption and future misery with certainty cannot be asked to supply any positive labor effort: the incentive constraint $u(0)+v(0)+$ $\beta w_{m} \geq u(0)+v(1-\hat{l})+\beta w_{m}$, reveals that misery is not incentive compatible for any recommended $l>0$ and that the only incentive-compatible allocation associated with forever repeated zero consumption is full leisure. Therefore, the exogenous lower bound on the stationary distribution is $\underline{w} \equiv(1-\beta)^{-1}\{u(0)+v(1)\}$.

The component planner associated with agents entitled to $\underline{w}$ can deliver this utility entitlement in two ways. He can either assign zero consumption and full leisure for all periods with cost $V(\underline{w})=0$, or, if it is less costly, an incentivecompatible positive labor supply and non-zero consumption together with an increased continuation utility in case of a high realization of output.

The planner will choose the first option if the technology is not productive enough for the incentive-compatible labor supply to repay the cost of capital input and consumption of the agent. Then the optimal, cost-minimizing allocations of continuation utility entitlements will drive all agents to $\underline{w}$ provided that the interest rate is lower than the time preference of the agents, $\rho=\frac{1-\beta}{\beta}$.

Lemma 2 If the incentive compatible allocation at $\underline{w}$ results in a positive current cost, $\int_{Y}\{c(\underline{w}, y)+(r+\delta) k-y\} P(d y \mid k(\underline{w}), l(\underline{w}))>0$, then the only feasible stationary distribution of utility entitlements is degenerate at $\underline{w}$ with interest rate $r \in(0, \rho)$.

For optimal labor supply policy $l(\underline{w})=0$ the continuation utility entitlement policy follows $V^{\prime}\left(w^{\prime}(\underline{w}, 0)\right)=(1+r) \beta V^{\prime}(\underline{w})$ and with $r \in(0, \rho)$ the lower bound $\underline{w}$ 
becomes an absorbing point. If $r=\rho$ the agents stay at their initial entitlements while if the interest rate is greater than the discount factor, all agents will be eventually driven to the exogenous upper bound. Neither equilibrium is feasible since there is positive consumption with zero output.

The stationary distribution of utility entitlements will exhibit mobility if the planner can choose the second, productive option, i.e., if the technology is sufficiently productive to repay the cost of capital input and the consumption reward for incentive-compatible labor supply $l(w)>0$. The endogeneity of the lower bound depends on the shape of the cost function. Denoting $w_{*} \in[\underline{w}, \bar{w}]$ as the minimum of the convex cost function, the lower bound will be endogenous at $w_{*}>\underline{w}$ if the cost function is decreasing in $w \in\left[\underline{w}, w_{*}\right)$. On the other hand, the lower bound will be exogenous at $w_{*}=\underline{w}$ if the cost function is non-decreasing in all $w \in[\underline{w}, \bar{w}]$. For example, a general-equilibrium extension of the numerical simulation in Phelan and Townsend (1991) would lead to a stationary distribution with an endogenous lower bound.

Whether the cost function is initially decreasing depends on the properties of the production and utility functions. The cost function decreases in $w \in\left[\underline{w}, w_{*}\right)$ if the one-period cost is also decreasing, which requires the incentive compatible labor supply and expected output to be increasing in the same interval. As before, the technology must be sufficiently productive to repay the cost of capital input and the consumption reward. The next Lemma shows that in the decreasing part of the cost function the planner can reduce his cost at the same time as making the agent better off by assigning a continuation utility in the non-decreasing part of the cost function, $w^{\prime}(w, y) \geq w_{*}$.

Lemma 3 If the current cost $\int_{Y}\{c(w, y)+(r+\delta) k-y\} P(d y \mid k(w), l(w))<0$ is decreasing in $w \in\left[\underline{w}, w_{*}\right)$, the planners' cost function $V(w)$ is decreasing in $w \in$ $\left[\underline{w}, w_{*}\right)$ and the optimal continuation utility policy assigns $w^{\prime}(w, y) \geq w_{*}$ for all $w \in\left[\underline{w}, w_{*}\right)$ and $y \in Y$.

For the either type (exogenous or endogenous) of the lower bound $w_{*}$, I will now establish the existence of an ergodic set with an endogenous upper bound. 


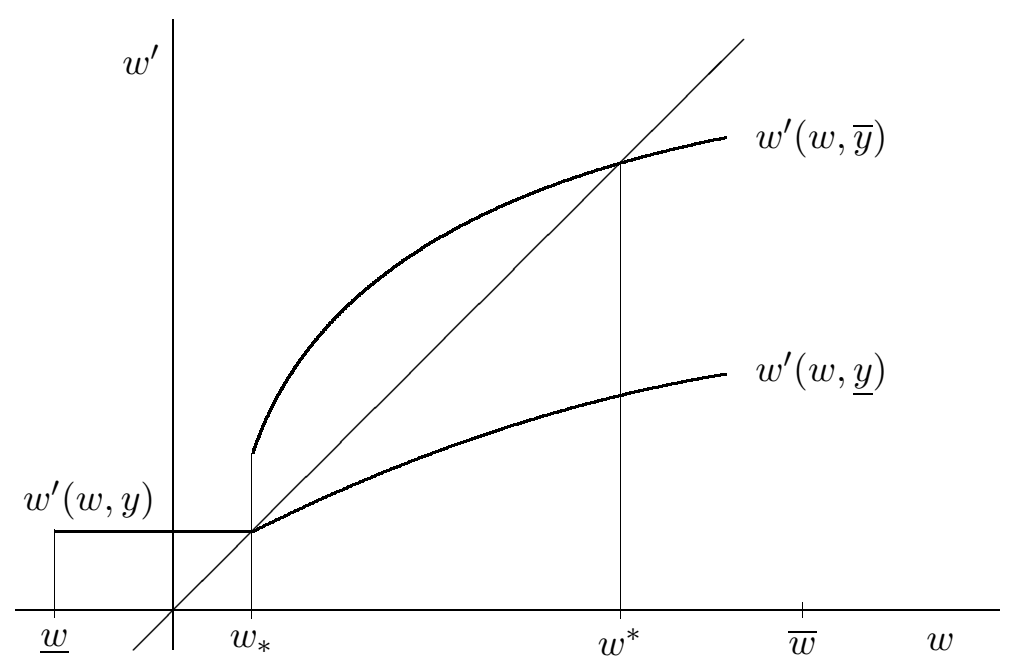

Figure 1: Example of continuation utility policy functions for high and low output realizations at interest rate $r \in(0, \rho)$ with endogenous ergodic set $\left[w_{*}, w^{*}\right]$.

Lemmas 4 and 5 analyze the behavior of continuation utility policies for $w \in$ $\left[w_{*}, \bar{w}\right]$. Without loss of generality I consider the highest and the lowest level of output realizations, $\bar{y}$ and $y(=0)$, respectively.

Lemma 4 For interest rate $r \in(0, \rho)$, all inputs $(k, l) \in K \times L$ and a low output realization $y \in Y$,

1. $w^{\prime}(w, \underline{y})=w_{*}$ for $w=w_{*} ;$ and

2. $w^{\prime}(w, y)<w$ for $w>w_{*}$.

Lemma 5 For interest rate $r \in(0, \rho)$, all inputs $(k, l) \in K \times L$ and a high output realization $\bar{y} \in Y$, there exists utility entitlement $w^{*} \in\left(w_{*}, \bar{w}\right)$ such that

1. $w^{\prime}(w, \bar{y})>w$ for $w<w^{*}$;

2. $w^{\prime}(w, \bar{y})=w$ for $w=w^{*}$; and

3. $w^{\prime}(w, \bar{y})<w$ for $w>w^{*}$.

If $r \geq \rho, w^{\prime}(w, \bar{y}) \geq w$ for all $w \in W$.

The continuation entitlement policies for an endogenous lower and upper bounds with interest rate $r \in(0, \rho)$ are illustrated in Figure 1. For such an interest 
rate and a sufficiently productive technology, there exists a non-degenerate invariant distribution of utility entitlements that satisfies the conditions of stationary recursive equilibria. For all $w \in\left(w_{*}, w^{*}\right)$, the planners punish agents with a low output realization by a lower continuation utility and reward agents with a high output realization by increasing their utility entitlement from tomorrow on. It is not cost efficient to punish agents below $w_{*}$ and reward them beyond $w^{*}$. These results follow from the component planner's intertemporal tradeoff of resources (see the Appendix for derivation),

$$
V_{r}^{\prime}\left(w^{\prime}(w, y)\right)=(1+r) \beta\left[V_{r}^{\prime}(w)+\xi(w)\left(1-\frac{P(y \mid k, \hat{l})}{P(y \mid k, l)}\right)\right],
$$

where the value function is convex, $(1+r) \beta<1$ if $r \in(0, \rho)$, the Lagrange multiplier $\xi(w)$ on the incentive constraint is positive, and the ratio $P(y \mid k, \hat{l}) / P(y \mid k, l)$ is a decreasing function of output for any downward deviation on labor effort $\hat{l} \in L$.

Theorem 1 If $\int_{Y}\left\{c\left(w_{*}, y\right)+(r+\delta) k-y\right\} P\left(d y \mid k\left(w_{*}\right), l\left(w_{*}\right)\right)<0$ and the interest rate $r \in(0, \rho)$, there exists a non-degenerate stationary distribution of utility entitlements in the ergodic set $\left[w_{*}, w^{*}\right]$ with an endogenous upper bound. If the cost function is initially decreasing in $w$, the lower bound is endogenous as well.

First, the analysis confirms the results of Kehoe and Levine (1993), Huggett (1997) and Lucas (1992) that in a private information economy the market clearing interest rate is less than the agents' time preference. If the component planner discounts the future more than the agents, i.e., if $r \geq \rho$, he tends to shift his costs into the future by promising higher continuation utility. On the other hand, if $r \in(0, \rho)$, the component planner cares about the future more than the agents and does not overuse future resources for current incentives.

Second, contrary to the private-information endowment economies, the lower bound of the ergodic set can be endogenous too. This result follows from the Pareto-improving allocations at the lowest levels of utility entitlements for an initially decreasing cost function. The only requirement is that the planners' cost function is initially decreasing in $w$ and the technology be sufficiently profitable 
to pay for the cost of capital input and current consumption of the agent. If the technology does not cover the cost of production and consumption of the agent, the only feasible stationary distribution is degenerate at the exogenous lower bound with interest rate again lower than the discount factor of the agents.

It remains to study the relationship between the outside transfers and invariant distribution $\psi_{r}$ of utility entitlements for different levels of interest rate. Since the policies for continuation utility determine the invariant distribution, the behavior of $\psi_{r}$ with respect to the interest rate depends on the relationship between $w^{\prime}(w, y)$ and interest rate $r$.

First, arguments in Atkeson and Lucas (1995) and Stokey, Lucas, and Prescott (1989) can be used to establish that $V_{r}$ and $w_{r}^{\prime}(w, y)$ vary continuously in the interest rate. Following Krueger (1999) I will show that the higher the interest rate, the more the component planners shift cost to the future by offering their agents a higher continuation utility in exchange for a lower level of current consumption.

Lemma 6 The optimal policy $w^{\prime}(w, y)$ is increasing and the optimal policy $c(w, y)$ is decreasing in $r$, respectively.

Because the invariant distribution is determined by the continuation utility policies, a higher interest rate leads to an invariant distribution with more mass on higher utility entitlements.

Lemma 7 If $r>\hat{r}$ then $\psi_{r} \geq \psi_{\hat{r}}$.

Finally, in numerical simulations one has to find a market-clearing interest rate. For that purpose define an aggregate deficit of all the component planners in one period in the steady state as

$$
\tau\left(\psi_{r}\right) \equiv \int_{W \times Y}\{c(w, y)+\delta k(w)-y\} P(d y \mid k(w), l(w)) \lambda_{r}(d w) .
$$

The last Lemma shows that the aggregate deficit is a decreasing function of the interest rate.

Lemma $8 \tau\left(\psi_{r}\right)$ is increasing in $r$. 
Thus during numerical simulations the iteration on interest rate proceeds according to the following algorithm: 1 . Guess an initial interest rate; 2. Find the optimal policies for all component planners and the invariant distribution of utility entitlements; 3. If the component planners spend more on consumption and investment than the agents produce $\left(\tau\left(\psi_{r}\right)>0\right)$, repeat with a lower interest rate (and vice versa for a surplus). Numerical simulations of different moral hazard economies are carried in Bohacek (2000).

\section{Conclusions}

This paper shows how to incorporate capital into private information economies using the concept of a financial intermediary, a 'capital planner'. Such a framework allows modeling general equilibrium economies with capital accumulation and private information without any restrictive assumptions on functional forms of production technology or preferences. An interesting extension could follow Fernandes and Phelan (2000) method of incorporating serially correlated productivity shocks into private information dynamic models.

Analysis of a closed economy with moral hazard and heterogeneous agents shows that with a sufficiently productive technology there is no need for imposing exogenous lower bound on utility entitlements in order to obtain a non-degenerate stationary distribution in a steady state. This feature can be used in analysis of distribution and allocation of resources in closed economies under different information constraints and contractual framework, including different efficiency levels of financial intermediation or various degrees of welfare state policies and social insurance provided by the government. 


\section{References}

Aiyagari, R. S. and F. Alvarez (1995). Stationary efficient distributions with private information: A tale of kings and slaves. Manuscript.

Atkeson, A. (1991). International lending with moral hazard and risk of repudiation. Econometrica 9, 1069-1089.

Atkeson, A. and R. E. Lucas, Jr. (1992). On efficient distribution with private information. Review of Economic Studies 59, 427-453.

Atkeson, A. and R. E. Lucas, Jr. (1995). Efficiency and equality in a simple model of efficient unemployment insurance. Journal of Economic Theory 66, 64-98.

Bohacek, R. (2000). The efficiency-equality tradeoff in welfare state economies. CERGE-EI Working Paper Series.

Fernandes, A. and C. Phelan (2000). A recursive formulation for repeated agency with history dependence. Journal of Economic Theory 91(2), 223-247.

Green, E. J. (1987). Lending and the smoothing of uninsurable income. In E. Prescott and N. Wallace (Eds.), Contractual Arrangements for Intertemporal Trade. Minneapolis: University of Minnesota Press.

Huggett, M. (1997). The one-sector growth model with idiosyncratic shocks: Steady states and dynamics. Journal of Monetary Economics 39, 385-403.

Jewitt, I. (1988). Justifying the first-order approach to principal-agent problem. Econometrica 56(5), 1177-1190.

Kehoe, T. J. and D. K. Levine (1993). Debt-constraint asset market. Review of Economic Studies 60, 865-888.

Khan, A. and B. Ravikumar (1997a). Enduring relationships in an economy with capital. Manuscript.

Khan, A. and B. Ravikumar (1997b). Growth and risk-sharing with private information. Manuscript.

Krueger, D. (1999). Risk sharing in economies with incomplete markets. University of Minnesota Working paper.

Lehnert, A., E. Ligon, and R. M. Townsend (1998). Liquidity constraints and incentive contracts. Manuscript.

Lucas, Jr., R. E. (1992). On efficiency and distribution. The Economic Journal (102), 233-247.

Phelan, C. (1995). Repeated moral hazard and one-sided commitment. Journal of Economic Theory 66, 488-506.

Phelan, C. and R. M. Townsend (1991). Computing multi-period, informationconstrained optima. Review of Economic Studies 58, 853-881.

Prescott, E. C. and R. M. Townsend (1984). Pareto optima and competitive equilibria with adverse selection and moral hazard. Econometrica 52(1), 21-45. 
Spear, E. and S. Srivastava (1987). On repeated moral hazard with discounting. Review of Economic Studies 55, 599-617.

Stokey, N. L., R. E. Lucas, Jr., and E. C. Prescott (1989). Recursive Methods in Economic Dynamics. Cambridge: Harvard University Press.

Thomas, J. and T. Worrall (1990). Income fluctuations and asymmetric information: An example of the repeated principal agent problem. Journal of Economic Theory $51,367-390$.

\section{Appendix}

\section{Proof of Proposition 1}

The arguments follow the appendix to Phelan and Townsend (1991). With lotteries, the problem is a minimization of a continuous function $V$ over a compact, non-empty set by applying the operator $T_{r}$. The minimum exists because the objective function is an integral over bounded continuous functions, defined on $K \times L \times Y \times C \times W^{\prime}$. $T_{r} V$ is continuous by the Theorem of the Maximum. Since $r>0$, the operator $T_{r}$ satisfies the hypotheses of Blackwell's theorem and is a contraction mapping of modulus $1 /(1+r)$. Spear and Srivastava (1987) show in Proposition 4.4 that the value function is strictly convex.

The proof of optimal conditional allocation $\pi\left(c, w^{\prime} \mid k, l, y ; w\right)=1$ for every $w \in W$ is based on Prescott and Townsend (1984). At the market-clearing interest rate $r$, the problem of a component planner, assigned to agent $w$, is to choose rewards in terms of consumption and continuation utility entitlement, $\left(c, w^{\prime}\right) \in C \times W$, conditional on a input-output combination $(k, l, y) \in K \times L \times Y$. The component planner's choice now becomes a probability measure $\pi(k, l, y, \cdot \mid w)$ which minimizes

$$
\begin{aligned}
V(w,(k, l)) & =\sum_{Y} P(y \mid k, l) . \\
\min _{\pi(k, l, y, \cdot \mid w)} & \sum_{C \times W^{\prime}} \pi\left(k, l, y, c, w^{\prime} \mid w\right)\left\{c+(r+\delta) k-y+\frac{1}{1+r} V\left(w^{\prime}\right)\right\},
\end{aligned}
$$

subject to the promise-keeping constraint,

$$
\sum_{Y} P(y \mid k, l) \sum_{C \times W^{\prime}} \pi\left(k, l, y, c, w^{\prime} \mid w\right)\left\{u(c)+v(1-l)+\beta w^{\prime}\right\}=w,
$$

the incentive constraint, for all $\hat{l} \in L$,

$$
\begin{aligned}
& \sum_{Y} P(y \mid k, l) \sum_{C \times W^{\prime}} \pi\left(k, l, y, c, w^{\prime} \mid w\right) \\
& \qquad\left\{u(c)+v(1-l)+\beta w^{\prime}-\frac{P(y \mid k, \hat{l})}{P(y \mid k, l)}\left[u(c)+v(1-\hat{l})+\beta w^{\prime}\right]\right\} \geq 0
\end{aligned}
$$


and the probability measure condition,

$$
\sum_{C \times W^{\prime}} \pi\left(k, l, y, c, w^{\prime} \mid w\right)=1 .
$$

Denote $\eta$ as the Lagrange multiplier on the promise keeping constraint (11), $\xi$ on the incentive constraint (12), and $\theta$ on the probability condition (13). Recall that the technology $P(y \mid k, l)>0$ is fixed for all combinations of inputs and outputs. The first order condition with respect to $\pi(k, l, y, \cdot \mid w)$ is:

$$
\begin{gathered}
c+(r+\delta) k-y+\frac{1}{1+r} V\left(w^{\prime}\right)-\left(u(c)+\beta w^{\prime}\right)\left[\eta+\xi\left(1-\frac{P(y \mid k, \hat{l})}{P(y \mid k, l)}\right)\right] \\
-\xi(v(1-l)-v(1-\hat{l})) \frac{P(y \mid k, \hat{l})}{P(y \mid k, l)}-\frac{\theta}{P(y \mid k, l)} \geq 0 .
\end{gathered}
$$

First note that the above condition must hold at equality for some $\left(c, w^{\prime}\right) \in C \times W$. If it did not, all probabilities $\pi\left(k, l, y, c, w^{\prime} \mid w\right)$ would be zero, violating the summation constraint (13).

Now analyze the left-hand side of (14), as a function of $\left(c, w^{\prime}\right)$. The Lagrange multipliers must be such that this function has a minimum at zero, at points where (14) holds at equality. Suppose that the set $C \times W$ is very large so that the maximal distance between any two points is arbitrarily small. Recall that the utility function is strictly concave, increasing function in both arguments and $V$ is strictly convex function. Define

$$
x \equiv \eta+\xi\left(1-\frac{P(y \mid k, \hat{l})}{P(y \mid k, l)}\right) .
$$

Then if $x \leq 0,1+(1+r)^{-1} V^{\prime}\left(w^{\prime}\right)-x\left(u^{\prime}(c)+\beta\right)>0$, since all terms but $x$ are positive. Then the left-hand side of (14) is strictly increasing function of $\left(c, w^{\prime}\right)$ within the nondecreasing part of the planners' cost function.

If $x>0$, the Hessian matrix for (14) is positive definite, $|H|=-(1+$ $r)^{-1} V_{r}^{\prime \prime}\left(w^{\prime}\right) x u^{\prime \prime}(c)>0$, and the left-hand side of (14) is strictly convex function of $\left(c, w^{\prime}\right)$. In both cases the left-hand side of (14) attains a minimum at a single combination of rewards $\left(c, w^{\prime}\right) \in C \times W$.

Therefore, for each pair of recommended inputs, the probability measure puts all mass on single combination of the rewards $\left(c, w^{\prime}\right) \in C \times W$ conditional on assigned inputs and realization of output. These points only depend on the utility entitlement level $w$, interest rate $r$, technology specification and parameters of the model.

Assuming interior solutions in a sufficiently large set $C \times W$, so that the allocations satisfy the appropriate conditions for maxima as if $C \times W$ were a continuum, it is possible to take the analogous first order conditions for (14). Using the envelope condition $V_{r}^{\prime}(w)=\eta$

$$
V_{r}^{\prime}\left(w^{\prime}(w, y)\right)=(1+r) \beta\left[V_{r}^{\prime}(w)+\xi\left(1-\frac{P(y \mid k, \hat{l})}{P(y \mid k, l)}\right)\right],
$$


and

$$
\frac{1}{u^{\prime}(c(w, y))}=V_{r}^{\prime}(w)+\xi\left(1-\frac{P(y \mid k, \hat{l})}{P(y \mid k, l)}\right)
$$

so that

$$
u^{\prime}(c(w, y)) V_{r}^{\prime}\left(w^{\prime}(w, y)\right)=(1+r) \beta .
$$

In case when the lowest labor supply is recommended to the agent, these conditions simplify to $u^{\prime}(c(w)) V_{r}^{\prime}(w)=1$ and $V_{r}^{\prime}\left(w^{\prime}(w)\right)=(1+r) \beta V_{r}^{\prime}(w)$.

To summarize, a component planner associated with agents entitled to $w \in W$ chooses input combination $(k, l) \in \arg \min _{\left(k^{\prime}, l^{\prime}\right) \in K \times L} V_{r}\left(w,\left(k^{\prime}, l^{\prime}\right)\right)$ with unique rewards $\left(c, w^{\prime}\right) \in C \times W$ for each realization of output $y \in Y$.

(Note: Even if the component planners ex ante randomized in inputs, the uniqueness of optimal $\left(c, w^{\prime}\right)$ for each pair of $(k, l)$ suffices for all proofs in the paper since the randomization results in a convex combination of rewards $\left(c, w^{\prime}\right)$.)

\section{Proof of Lemma 1}

The combination of the first order conditions for consumption (15) and continuation utility (16) obtains $u^{\prime}(c(w, y)) V_{r}^{\prime}\left(w^{\prime}(w, y)\right)=(1+r) \beta$. In the non-decreasing part of the cost function $u^{\prime}>0$ and concave while $V_{r}^{\prime}>0$ and convex, so that $c(w, y)$ and $w^{\prime}(w, y)$ move in the same direction as $w$ changes. Suppose, as a contradiction, that both $c(w, y)$ and $w^{\prime}(w, y)$ are decreasing functions of $w$, so that for $\underline{w} \leq \hat{w}<w \leq \bar{w}, c(\hat{w}, y)>c(w, y)$ and $w^{\prime}(\hat{w}, y)>w^{\prime}(w, y)$ for all $y \in Y$. Then from the promise-keeping constraint

$$
w=u(c(w, y))+v(1-l)+\beta w^{\prime}(w, y)<u(c(\hat{w}, y))+v(1-l)+\beta w^{\prime}(\hat{w}, y)=\hat{w}
$$

which contradicts the initial assumption that $\hat{w}<w$. Therefore, $c(w, y)$ and $w^{\prime}(w, y)$ are both increasing functions of $w$ for all $y \in Y$.

To prove the second claim of the Lemma, note first that for $l=0$, there is no incentive compatibility constraint and the first order conditions become

$$
u^{\prime}(c(w, y)) V_{r}^{\prime}(w)=1 \text { and } V_{r}^{\prime}\left(w^{\prime}(w, y)\right)=(1+r) \beta V_{r}^{\prime}(w),
$$

respectively, with $c(w, y)$ and $w^{\prime}(w, y)$ independent of output realization.

For $l>0$, the incentive compatibility constraint binds and the first order conditions are as in the proof of Proposition 1, equations (15) and (16), respectively. Since the utility is increasing and concave in consumption and increasing in leisure, $\xi(w)>0$ by Lemma 1 in Jewitt (1988). Recall that $P(y \mid k, \hat{l}) / P(y \mid k, l)$ ratio is a decreasing function of $y$ for any deviating strategy $\hat{l}<l$ at the given level of recommended inputs. Then for all $w \in W, w^{\prime}(w, \bar{y})>w^{\prime}(w, \underline{y})$ and $c(w, \bar{y})>c(w, \underline{y})$ for all $w \in W$ and $\underline{y}, \bar{y} \in Y$, such that $\bar{y}>\underline{y}$. 


\section{Proof of Lemma 2}

If the cost-minimizing optimal allocation at $\underline{w}$ assigns $l(\underline{w})=0$, there is no consumption and no output. Then for $r \in(0, \rho]$ the first-order intertemporal condition $V^{\prime}\left(w^{\prime}(\underline{w}, 0)\right)=$ $(1+r) \beta V^{\prime}(\underline{w})$ implies that at the lower bound $w^{\prime}(\underline{w}, 0)=\underline{w}$ and $V(\underline{w})=0$. For a cost function non-decreasing in $w \in W$ any other allocation would lead to a higher current and future costs and would not be optimal.

Then $\underline{w}$ is an absorbing point rather than a reflecting barrier for $r \in(0, \rho)$ and the distribution puts the mass of all agents at $\underline{w}$ since for $l(w)=0$ the continuation utilities $w^{\prime}(w, 0)<w$ for all $w \in W$ and $r \in(0, \rho)$; for $l(w)>0$ the probability of low output, and therefore, decreasing continuation utility, is positive for all $w \in W$. This equilibrium is feasible since all agents neither produce nor consume. For interest rate $r \geq \rho$ the continuation utility policies would be nondecreasing in $w$ and the stationary distribution would exhibit no mobility at utility entitlements with positive consumption and zero output (see Lemmas 4 and 5 for details).

\section{Proof of Lemma 3}

First consider the optimal continuation policy function for $w \in\left[\underline{w}, w_{*}\right)$ provided that the cost function is decreasing with a minimum at $w_{*}$. Consider $l \in(0,1]$ and, without loss of generality, $\underline{y}, \bar{y} \in Y$ such that $\bar{y}>\underline{y}$. For $w \in\left[\underline{w}, w_{*}\right)$, denote $\left\{w_{*}^{\prime}(w, y), c_{*}(w, y), V_{*}(w)\right\}$ as allocations and value associated with continuation policies $w_{*}^{\prime}(w, \bar{y}) \geq w_{*}^{\prime}(w, \underline{y})=w_{*}$. Correspondingly, denote $\left\{w^{\prime}(w, y), c(w, y), V(w)\right\}$ as allocations and value associated with continuation policies $w^{\prime}(w, \underline{y})<w_{*} \leq w^{\prime}(w, \bar{y})$. Let both allocations satisfy the promise keeping and incentive constraints.

Rewrite the intertemporal first order condition as $V_{r}^{\prime}\left(w^{\prime}(w, y)\right)=(1+r) \beta\left[V_{r}^{\prime}(w)+\right.$ $\xi\left(P_{l}(y \mid k, l) / P(y \mid k, l)\right]$ where $P_{l}(y \mid k, l)=\partial P(y \mid k, l) / \partial l$. For a given $l(w)$, integration with respect to $P(d y \mid k(w), l(w))$ delivers $\int_{Y} V\left(w^{\prime}(w, y)\right) P(d y \mid k(w), l(w))=$ $(1+r) \beta V^{\prime}(w)$ for all $w \in W$. Therefore $\int_{Y} w_{*}^{\prime}(w, y) P(d y \mid k(w), l(w))=$ $\int_{Y} w^{\prime}(w, y) P(d y \mid k(w), l(w))$ and $w^{\prime}(w, \bar{y})>w_{*}^{\prime}(w, \bar{y})$. The promise keeping constraint implies that $\int_{Y} c_{*}(w, y) P(d y \mid k(w), l(w))=\int_{Y} c(w, y) P(d y \mid k(w), l(w))$. Then for continuation utility policies $w^{\prime}(w, \underline{y})<w_{*}=w_{*}^{\prime}(w, \underline{y}) \leq w_{*}^{\prime}(w, \bar{y})<w^{\prime}(w, \bar{y})$ the strictly convex cost function with minimum at $w_{*}$ implies that $V\left(w_{*}\right) \leq$ $\int_{Y} V\left(w_{*}^{\prime}(w, y)\right) P(d y \mid k(w), l(w))<\int_{Y} V\left(w^{\prime}(w, y)\right) P(d y \mid k(w), l(w))$. Then $V_{*}(w)<$ $V(w)$ and the cost-minimizing allocations assign $w^{\prime}(w, y) \geq w_{*}$ for any $w \in\left[\underline{w}, w_{*}\right)$, all $y \in Y$ and any positive labor supply $l \in(0,1]$. For $l=0$ the continuation utility and consumption rewards do not depend on output and similar arguments lead to optimal $w^{\prime}(w, y)=w^{\prime}(w)=w_{*}$ for all $w \in\left[\underline{w}, w_{*}\right)$.

With consumption policy function $c(w, y)$ increasing, convex in $w$ for all $y \in Y$ and production function increasing, concave in $l$, the one-period cost $\int_{Y}\{c(w, y)+(r+\delta) k-y\} P(d y \mid k(w), l(w))$ is decreasing, convex function only for an increasing $l(w)$ in $w \in\left[\underline{w}, w_{*}\right)$ and increasing expected output. If planners can also achieve current negative cost, $\int_{Y}\{c(w, y)+(r+\delta) k-y\} P(d y \mid k(w), l(w))<0$, the cost function is also decreasing in $w \in\left[\underline{w}, w_{*}\right)$. 


\section{Proof of Lemma 4}

It is not possible to assign $w^{\prime}(w, y)<w_{*}$ if the lower bound is exogenously set at $w_{*}$. Lemma 3 proved that it is not optimal to assign a continuation utility lower than the endogenous lower bound $w_{*}$.

To study optimal continuation utility entitlement policies for the strictly increasing part of the cost function at $w \in\left(w_{*}, \bar{w}\right]$ when a low output is realized, consider first the case when $l=0$. There is no incentive problem and the first order intertemporal condition equals $V_{r}^{\prime}\left(w^{\prime}(w, y)\right)=(1+r) \beta V_{r}^{\prime}(w)$. Then for $\left.V_{r}^{\prime}(w)>0, w^{\prime}(w, y)\right) \geq w$ if $r \geq \rho$ and $\left.w^{\prime}(w, y)\right)<w$ if $r \in(0, \rho)$. In case that $l>0$ the incentive constraint binds and $\xi(w)>0$. Then the intertemporal first order condition is

$$
V_{r}^{\prime}\left(w^{\prime}(w, \underline{y})\right)=(1+r) \beta\left[V_{r}^{\prime}(w)+\xi(w)\left(1-\frac{P(\underline{y} \mid k, \hat{l})}{P(\underline{y} \mid k, l)}\right)\right] .
$$

When $y=\underline{y}$, the ratio $P(\underline{y} \mid k, \hat{l}) / P(\underline{y} \mid k, l)>1$ for any downward deviation $\hat{l} \in L$. Hence the term multiplying the positive Lagrange multiplier is negative and for $r \in(0, \rho)$ the continuation utility entitlement decreases, $w^{\prime}(w, y)<w$ for all $w>w_{*}$.

\section{Proof of Lemma 5}

First, consider the case when $l=0$. Then the output is zero with certainty and the first order intertemporal condition is $V_{r}^{\prime}\left(w^{\prime}(w, \bar{y})\right)=(1+r) \beta V_{r}^{\prime}(w)$, so that for the increasing part of the cost function, $w^{\prime}(w, \bar{y}) \geq w$ if $r \geq \rho$ and $w^{\prime}(w, \bar{y})<w$ if $r \in(0, \rho)$.

In case that $l>0$ the incentive constraint binds and $\xi(w)>0$. For the high output level the ratio $P(\bar{y} \mid k, \hat{l}) / P(\bar{y} \mid k, l)<1$ for any downward deviation $\hat{l} \in L$. Recall the definition of the highest utility entitlement, $\bar{w} \equiv u(\bar{c})+v(1)+\beta \bar{w}^{\prime}$. Since for any $y \in Y$, $w^{\prime}(w, y)<w$ for all $w \in W$ when $l=0$ and $r \in(0, \rho)$, the highest possible utility entitlement is not sustainable if $l=0$ and is not attainable if $l>0$.

In the latter case suppose that an agent begins with some arbitrary initial entitlement $w<\bar{w}$, corresponding to some $c<\bar{c}$ and $l>0$. Since the highest continuation utility $\bar{w}$ is not attainable, he can be promised $w^{\prime}=\bar{w}-\epsilon$ for some $\epsilon>0$, i.e., he begins with a promised utility $w=u(c)+v(1-l)+\beta(\bar{w}-\epsilon)$. The component planner's first order intertemporal condition (15) becomes

$$
V_{r}^{\prime}(\bar{w}-\epsilon, \bar{y})=(1+r) \beta\left[V_{r}^{\prime}(w)+\xi(w)\left(1-\frac{P(\bar{y} \mid k, \hat{l})}{P(\bar{y} \mid k, l)}\right)\right] .
$$

For establishing the endogenous upper bound on utility entitlement, suppose that the agent's utility entitlement increased to $w^{\prime}(w, \bar{y})=\bar{w}-\epsilon>w$. The argument can be repeated for a sequence of periods until an entitlement $w=\bar{w}-\epsilon^{*}$, with $\epsilon^{*}>0$, is reached at which $w^{\prime}=\bar{w}-\epsilon^{*}=w$, i.e., $w=\bar{w}-\epsilon^{*}=u(c)+v(1-l)+\beta\left(\bar{w}-\epsilon^{*}\right)$.

Since $c \in C$ and $l \in(0,1]$ were arbitrary optimal allocations, for $r \in(0, \rho)$ there 
exists a utility entitlement $w^{*} \in\left(w_{*}, \bar{w}\right)$ for which $w^{\prime}\left(w^{*}, \bar{y}\right)=w^{*}$, i.e.,

$$
V_{r}^{\prime}\left(w^{*}\left(w^{*}, \bar{y}\right)\right)=(1+r) \beta\left[V_{r}^{\prime}\left(w^{*}\right)+\xi\left(w^{*}\right)\left(1-\frac{P(\bar{y} \mid k, \hat{l})}{P(\bar{y} \mid k, l)}\right)\right] .
$$

For utility entitlements $w<w^{*}$ the continuation utility entitlement increases to $w^{\prime}\left(w^{*}, \bar{y}\right)>w^{*}$, and for $w>w^{*}$ decreases $w^{\prime}(w, \bar{y})<w$. When $r \geq \rho, w^{\prime}(w, \bar{y}) \geq w$ for all $w \in W$.

\section{Proof of Theorem 1}

See Lemmas 2 - 5. For the proof of convergence to the unique invariant probability measure $\lambda_{r}$ from an arbitrary probability measure $\lambda_{0}$, see Theorem 3.2 and Lemma 11.11 in Stokey, Lucas, and Prescott (1989).

\section{Proof of Lemma 6}

Let $r>\hat{r}$ and show $w_{r}^{\prime}(w, y) \geq w_{\hat{r}}^{\prime}(w, y)$ and $c_{r}(w, y) \leq c_{\hat{r}}(w, y)$. Define $\left\{V^{n}\right\}_{n=1}^{\infty}$ by $V^{n}=\left(T_{\hat{r}}^{n}\right) V_{r}$. Let $\left(c_{n}(w, y), w_{n}^{\prime}(w, y)\right)$ and $\left(c_{r}(w, y), w_{r}^{\prime}(w, y)\right)$ be the optimal policies associated with $V^{n}$ and $V_{r}$, respectively, where $V^{n}(w)$ is defined as

$$
V^{n}(w)=\int_{Y}\left\{c_{n}(w, y)+(r+\delta) k_{n}(w)-y+\frac{1}{1+r} V^{n-1}\left(w_{n}^{\prime}(w, y)\right)\right\} P\left(d y \mid k_{n}(w), l_{n}(w)\right) .
$$

Since $\left\{V^{n}, w_{n}^{\prime}(w, y)\right\}_{n=1}^{\infty}$ uniformly converges to $\left(V_{\hat{r}}, w_{\hat{r}}^{\prime}(w, y)\right)$, it is sufficient to prove that

$$
w_{r}^{\prime}(w, y) \geq w_{n}^{\prime}(w, y) \text { and } c_{r}(w, y) \leq c_{n}(w, y) .
$$

First, let $n=1, w \in\left[\underline{w}, w^{*}\right]$, and assume as a contradiction that for all $y \in$ $Y, w_{r}^{\prime}(w, y) \leq w_{1}^{\prime}(w, y)$. From the first order conditions,

$$
V_{r}^{\prime}\left(w_{1}^{\prime}(w, y)\right)=(1+\hat{r}) \beta \frac{1}{u^{\prime}\left(c_{1}(w, y)\right)} \text { and } V_{r}^{\prime}\left(w_{r}^{\prime}(w, y)\right) \geq(1+r) \beta \frac{1}{u^{\prime}\left(c_{r}(w, y)\right)} .
$$

The strict convexity of $V_{r}$, together with the assumption that $r>\hat{r}$ and $w_{r}^{\prime}(w, y) \leq$ $w_{1}^{\prime}(w, y)$, imply that $V_{r}^{\prime}\left(w_{1}^{\prime}(w, y)\right)>V_{r}^{\prime}\left(w_{r}^{\prime}(w, y)\right)$ and $c_{1}(w, y)>c_{r}(w, y)$.

However, from the promise-keeping constraint,

$$
w=\int_{Y}\left\{u(c(w, y))+v(1-l(w))+\beta w^{\prime}(w, y)\right\} P(d y \mid k(w), l(w)),
$$

there must exist a $\tilde{y} \in Y$ such that $w_{r}^{\prime}(w, \tilde{y})>w_{1}^{\prime}(w, \tilde{y})$. Then from the first order conditions,

$$
V_{r}^{\prime}\left(w_{r}^{\prime}(w, \tilde{y})\right)=(1+r) \beta \frac{1}{u^{\prime}\left(c_{r}(w, \tilde{y})\right)}<(1+\hat{r}) \beta \frac{1}{u^{\prime}\left(c_{1}(w, \tilde{y})\right)}=V_{r}^{\prime}\left(w_{1}^{\prime}(w, \tilde{y})\right),
$$


which implies $w_{r}^{\prime}(w, \tilde{y})<w_{1}^{\prime}(w, \tilde{y})$, a contradiction. Therefore, $w_{r}^{\prime}(w, y) \geq w_{1}^{\prime}(w, y)$ for all $y \in Y$. The promise keeping constraint implies $c_{r}(w, y) \leq c_{1}(w, y)$, for all $y \in Y$. The envelope condition yields $(1+r) V_{r}^{\prime}(w) \geq(1+\hat{r}) V_{1}^{\prime}(w)$.

Second, suppose that (19) hold for $n-1$. I want to show that the same is true also for $n$. By contradiction, suppose that for all $y \in Y, w_{r}^{\prime}(w, y) \leq w_{n}^{\prime}(w, y)$. Again, from the first order conditions,

$$
\left(V^{n-1}\right)^{\prime}\left(w_{n}^{\prime}(w, y)\right)=(1+\hat{r}) \beta \frac{1}{u^{\prime}\left(c_{n}(w, y)\right)}, \text { and } V_{r}^{\prime}\left(w_{r}^{\prime}(w, y)\right) \geq(1+r) \beta \frac{1}{u^{\prime}\left(c_{r}(w, y)\right)} .
$$

Since $V^{n-1}$ and $V_{r}$ are convex, together with the assumption that $r>\hat{r}$ and $w_{r}^{\prime}(w, y) \leq$ $w_{n}^{\prime}(w, y)$, it follows that $c_{n}(w, y)>c_{r}(w, y)$. Again, by the promise-keeping constraint, there must exist a $\tilde{y} \in Y$ such that $w_{r}^{\prime}(w, \tilde{y})>w_{n}^{\prime}(w, \tilde{y})$. Then from the envelope condition

$$
\left(V^{n}\right)^{\prime}(w)=\frac{1}{\beta(1+\hat{r})}\left(V^{n-1}\right)^{\prime}\left(w_{n}^{\prime}(w, \tilde{y})\right)<\frac{1}{\beta(1+r)} V_{r}^{\prime}\left(w_{r}^{\prime}(w, \tilde{y})\right)=V_{r}^{\prime}(w),
$$

which implies $w_{r}^{\prime}(w, \tilde{y})<w_{n}^{\prime}(w, \tilde{y})$, a contradiction. Therefore, $w_{r}^{\prime}(w, y) \geq w_{n}^{\prime}(w, y)$ for all $y \in Y$. The promise keeping constraint implies $c_{r}(w, y) \leq c_{n}(w, y)$, for all $y \in Y$.

\section{Proof of Lemma 7}

Define the sequence $\left\{\psi_{n}\right\}_{n=1}^{\infty}$ by $\psi_{n}=\left(\hat{T}_{\hat{r}}\right)^{n} \psi_{r}$ and prove by induction that for each $n \geq 1$ and each $y \in Y, \psi_{r}$ stochastically dominates $\psi_{n}$. Since by $\left\{\psi_{n}\right\}_{n=1}^{\infty}$ converges to $\left\{\psi_{\hat{r}}\right\}_{n=1}^{\infty}$ in total variation. Define $\psi_{n}^{y}$ as the distribution function associated with $\psi_{n}$ by $\psi_{n}^{y}: W \rightarrow[0,1]$, where $\psi_{n}^{y}(w)=\psi_{n}([\underline{w}, w])$ for all $y \in Y$. It is sufficient to prove that for all $w \in W, \psi_{r}^{y}(w) \leq \psi_{n}^{y}(w)$ for all $y \in Y$.

First, let $n=1$. By definition, $\psi_{1}=\left(\hat{T}_{\hat{r}}\right) \psi_{r}$ and $\psi_{r}=\left(\hat{T}_{r}\right) \psi_{r}$. Fix an arbitrary $y \in Y$ and $w \in W$. Then

$$
\psi_{r}^{y}(w)=\int_{\left\{z \in W \mid w_{r}^{\prime}(z, y) \leq w\right\}} \psi_{r}(d z) \leq \int_{\left\{z \in W \mid w_{\hat{r}}^{\prime}(z, y) \leq w\right\}} \psi_{r}(d z)=\psi_{\hat{r}}^{y}(w),
$$

where the inequality is due to the fact that $w_{r}^{\prime}(w, y) \geq w_{\hat{r}}^{\prime}(w, y)$ for all $w \in W$.

Second, suppose that $\psi_{r}^{y}(w) \leq \psi_{n-1}^{y}(w)$, for all $w \in W$ and $y \in Y$ and show that it is also true for $n$. Again,

$$
\psi_{r}^{y}(w)=\int_{\left\{z \in W \mid w_{r}^{\prime}(z, y) \leq w\right\}} \psi_{r}(d z) \leq \int_{\bar{y} \in Y} P\left(d \bar{y} \mid k\left(z_{r}\right), l\left(z_{r}\right)\right) \psi_{r}^{\bar{y}}\left(z_{r}\right),
$$

where $z_{r}=\max \left\{z \in W \mid w_{r}^{\prime}(z, y) \leq w\right\}$ and the equality follows from $w_{r}^{\prime}(w, y)$ being continuous and increasing in its first argument. Similarly, $\psi_{n}^{y}(w)=$ $\int_{\bar{y} \in Y} P\left(d \bar{y} \mid k\left(z_{n}\right), l\left(z_{n}\right)\right) \psi_{n-1}^{\bar{y}}\left(z_{n}\right)$, with $z_{n}=\max \left\{z \in W \mid w_{\hat{r}}^{\prime}(z, y) \leq w\right\}$.

Lemma 6 implies that $z_{r} \leq z_{n}$. Then from the induction hypothesis it follows that for all $\bar{y} \in Y, \psi_{r}^{\bar{y}}\left(z_{r}\right) \leq \psi_{n-1}^{\bar{y}}\left(z_{n}\right)$ and, therefore, $\psi_{r}^{y}(w) \leq \psi_{n}^{y}(w)$. 


\section{Proof of Lemma 8}

By definition and by $\psi_{r}$ being an invariant distribution,

$$
\begin{aligned}
\tau\left(\psi_{r}\right) & =\int_{W \times Y}\{c(w, y)+\delta k(w)-y\} P(d y \mid k(w), l(w)) \psi_{r}(d w) \\
& =\int_{W \times Y}\{c(w, y)+(r+\delta) k(w)-y\} P(d y \mid k(w), l(w)) \psi_{r}(d w) \\
& =\frac{1+r}{r} \int_{W} V_{r}(w) \psi_{r}(d w) .
\end{aligned}
$$

For $r>\hat{r}$, I need to prove that $\int_{W} V_{r}(w) \psi_{r}(d w) \geq \int_{W} V_{\hat{r}}(w) \psi_{\hat{r}}(d w)$. Since $\psi_{r}$ stochastically dominates $\psi_{\hat{r}}$ and $V_{r}$ is strictly increasing, $\int_{W} V_{\hat{r}}(w) \psi_{r}(d w) \geq \int_{W} V_{\hat{r}}(w) \psi_{\hat{r}}(d w)$, it is enough to show that $\int_{W} V_{r}(w) \psi_{r}(d w) \geq \int_{W} V_{\hat{r}}(w) \psi_{r}(d w)$. Define $\left\{V^{n}\right\}_{n=1}^{\infty}$ by $V^{n}=\left(T_{\hat{r}}^{n}\right) V_{r}$. From proofs above, the sequence $\left\{V^{n}\right\}_{n=1}^{\infty}$ converges uniformly to $V_{\hat{r}}$ and it is sufficient to prove that for $n \geq 1$,

$$
\int_{W} V_{r}(w) \psi_{r}(d w) \geq \int_{W} V^{n}(w) \psi_{r}(d w) .
$$

Also let $\left\{k_{n}(w), l_{n}(w), c_{n}(w, y), w_{n}^{\prime}(w, y)\right\}$ and $\left\{k_{r}(w), l_{r}(w), c_{r}(w, y), w_{r}^{\prime}(w, y)\right\}$ be the optimal policies associated with $\left\{V^{n}\right\}_{n=1}^{\infty}$ and $V_{r}$, respectively.

First, let $n=1$. By definition, $V^{1}=T_{\hat{r}} V_{r}$ and

$$
\begin{aligned}
V^{1}(w) & =\int_{Y}\left\{c_{1}(w, y)+(\hat{r}+\delta) k_{1}(w)-y+\frac{1}{1+\hat{r}} V_{r}\left(w_{1}^{\prime}(w, y)\right)\right\} P\left(d y \mid k_{1}(w), l_{1}(w)\right) \\
& \leq \int_{Y}\left\{c_{r}(w, y)+(\hat{r}+\delta) k_{r}(w)-y+\frac{1}{1+\hat{r}} V_{r}\left(w_{r}^{\prime}(w, y)\right)\right\} P\left(d y \mid k_{r}(w), l_{r}(w)\right),
\end{aligned}
$$

by the principle of optimality. Integrating with respect to $\psi_{r}$ yields

$$
\begin{aligned}
& \int_{W} V^{1}(w) \psi_{r}(d w)=\int_{W \times Y}\left\{c_{1}(w, y)+(\hat{r}+\delta) k_{1}(w)-y+\frac{1}{1+\hat{r}} V_{r}\left(w_{1}^{\prime}(w, y)\right)\right\} . \\
& \text { · } P\left(d y \mid k_{1}(w), l_{1}(w)\right) \psi_{r}(d w) \\
& \leq \int_{W \times Y}\left\{c_{r}(w, y)+(\hat{r}+\delta) k_{r}(w)-y+\frac{1}{1+\hat{r}} V_{r}\left(w_{r}^{\prime}(w, y)\right)\right\} . \\
& \cdot P\left(d y \mid k_{r}(w), l_{r}(w)\right) \psi_{r}(d w) \\
& =\int_{W} V_{r}(w) \psi_{r}(d w)
\end{aligned}
$$

using (20).

Second, suppose that $\int_{W} V_{r}(w) \psi_{r}(d w) \geq \int_{W} V^{n-1}(w) \psi_{r}(d w)$. To show that the same is true for $n$, use $V^{n}=T_{\hat{r}} V^{n-1}$ and

$$
V^{n}(w)=\int_{Y}\left\{c_{n}(w, y)+(\hat{r}+\delta) k_{n}(w)-y+\frac{1}{1+\hat{r}} V^{n-1}\left(w_{n}^{\prime}(w, y)\right)\right\} .
$$




$$
\begin{gathered}
\cdot P\left(d y \mid k_{n}(w), l_{n}(w)\right) \\
\leq \int_{Y}\left\{c_{r}(w, y)+(\hat{r}+\delta) k_{r}(w)-y+\frac{1}{1+\hat{r}} V^{n-1}\left(w_{r}^{\prime}(w, y)\right)\right\} \\
\cdot P\left(d y \mid k_{r}(w), l_{r}(w)\right)
\end{gathered}
$$

using again the principle of optimality. Integrating as above with respect to $\psi_{r}$ obtains

$$
\begin{aligned}
\int_{W} V^{n}(w) \psi_{r}(d w) & =\int_{W \times Y}\left\{c_{n}(w, y)+(\hat{r}+\delta) k_{n}(w)-y+\frac{1}{1+\hat{r}} V^{n-1}\left(w_{n}^{\prime}(w, y)\right)\right\} \\
& \leq \int_{W \times Y}\left\{c_{r}\left(d y \mid k_{n}(w), l_{n}(w)\right) \psi_{r}(d w)\right. \\
& =\int_{W \times Y}\left\{\frac{\hat{r}}{1+\hat{r}} V_{r}(w, y) k_{r}(w)-y+\frac{1}{1+\hat{r}} V^{n-1}\left(w_{r}^{\prime}(w, y)\right)\right\} \\
& \leq \int_{W \times Y}\left\{\frac{1}{1+\hat{r}} V^{n-1}\left(w_{r}^{\prime}(w, y)\right)\right\} \\
& =\int_{W} V_{r}(w) \psi_{r}(w, y)+\frac{1}{1+\hat{r}} V_{r}\left(w_{r}(d w),\right.
\end{aligned}
$$

using (20) and the induction hypothesis for the last inequality. 